\title{
Echocardiographic characteristics of male athletes of different age
}

\author{
G Pavlik, Z Olexó, P Osváth, Z Sidó, R Frenkl
}

\begin{abstract}
Two dimensionally guided $M$ mode and Doppler echocardiographic data for 578 male subjects (106 non-athletic and 472 athletes) were analysed from two aspects: $(a)$ in the young adult category (19-30 years of age), competitors in different groups of sports were studied; $(b)$ in the different age groups (children, 10-14 years; adolescent juniors, 15-18 years; young adults, 19-30 years; adults, 31-44 years; older adults $45-60$ years), data for athletes and non-athletes were compared. Morphological variables were related to body size by indices in which the exponents of the numerator and denominator were matched. Morphological signs of athletic heart were most consistently evident in the left ventricular muscle mass: in the young adult group, the highest values were seen in the endurance athletes, followed by the ball game players, sprinters/jumpers, and power athletes. A thicker muscular wall was the main reason for this hypertrophy. Internal diameter was only increased in the endurance athletes, and this increase was more evident in the younger groups. The E/A quotient (ratio of peak velocity during early and late diastole) indicated more effective diastolic function in the endurance athletes. The values for E/A quotient also suggested that regular physical activity at an older age may protect against age dependent impairment of diastolic function.

(Br f Sports Med 2001;35:95-99)
\end{abstract}

Keywords: echocardiography; heart; athletic heart; age; male athletes

Budapest, Hungary

G Pavlik

Z Olexó

P Osváth

R Frenkl

National Institute for Sports Medicine,

Department of

Conditioning and

Internal Medicine,

Budapest, Hungary

Z Sidó

Correspondence to:

Professor Pavlik, Semmelweis University, Faculty of

Physical Education and

Sports Sciences, Department

of Health Sciences and

Sports Medicine, H-1123,

Budapest, Alkotás u. 44.

pavlik@mail.hupe.hu

Accepted 29 January 2001

When comparing subjects of differing body size, it is essential to relate echocardiographic data to some body dimension. In our recent studies, ${ }^{12}$ we noticed that the most often used method, relation to body surface area (BSA), contains an inherent error arising from the difference in the exponents of the numerator and denominator. Similar observations have recently been reported by others. ${ }^{34}$

The denominator (BSA in $\mathrm{m}^{2}$ ) is always on the second power. When the exponent of the numerator is one (wall thickness, diameter in $\mathrm{mm}$ ), indices become disproportionately smaller with growing body size. On the other hand, when the exponent of the numerator is three (muscle mass, volumes in $\mathrm{g}$ or $\mathrm{ml}$ or $\mathrm{cm}^{3}$ ) indices appear greater with growing body size.
To avoid such spurious trends, we attempted to use indices in which power terms match. ${ }^{12}$ Preferring to keep the relation to BSA, we suggested that linear variables are related to the square root of BSA, with volumes and weights related to the cube of the square root of BSA. These indices did not show any correlation with body measures, so it became possible to compare data for subjects of different age, body size, or weight. Similar suggestions have recently been made by others. ${ }^{3}{ }^{4}$

In this study, these modified indices were used to analyse the echocardiographic results of a large number of male athletes and non-athletes. The results are discussed from two aspects. In the young adult athletes, the effects of their different sports are investigated; data for power athletes, sprinters/jumpers, ball game players, and endurance athletes are compared with each other and with data for young adult non-athletic subjects. A comparison is also made across the ages: from childhood to older age, data on athletes are compared with those on non-athletic healthy subjects.

As the main morphological characteristic of athletic heart-that is, myocardial hypertrophy - can also occur in several pathological states, training induced morphological modifications should be considered together with some functional parameters. In addition to the morphological variables, resting heart rate and E/A quotient - that is, the ratio of early and late transmitral flow velocity-will be given. The latter is a sensitive indicator of diastolic function - that is, left ventricular compliance. A decrease in the quotient unambiguously indicates an impairment of the diastolic function as the result of either advanced age $e^{5-7}$ or some pathological event. ${ }^{8-10}$

\section{Subjects and methods}

Table 1 gives some basic characteristics of the subjects. They were separated into five age groups as follows: children, 10-14 years; adolescents/juniors, 15-18 years; young adults in the age range at which most top class competition occurs, 19-30 years; adults, 31-44 years; older adults, $45-60$ years.

The controls were healthy males of comparable age who were not taking any medication and had no history of cardiac disease. The athletes were competitors of variable ability.

The child athletes were soccer players and swimmers who performed 8-10 hours of physical training a week. Adolescent and junior athletes were top level middle distance and long distance runners, competitive cyclists, triathletes, waterpolo and basketball players, and weight lifters. 
Table 1 Age, body surface area (BSA), and resting heart rate (HR) of the subjects

\begin{tabular}{llllll}
\hline Group & $N$ & $N^{\prime}$ & Age (years) & BSA $\left(\mathrm{m}^{2}\right)$ & HR (beats/min) \\
\hline CHLDc & 13 & 12 & $12.38(1.04)$ & $1.34(0.23)$ & $87.11(14.35)$ \\
CHLDa & 88 & 40 & $11.76(1.15)$ & $1.30(0.17)$ & $72.03(11.92) \ddagger$ \\
AJc & 10 & 10 & $17.40(0.84)$ & $1.94(0.18)$ & $71.75(8.99)$ \\
AJa & 77 & 32 & $16.53(1.10)$ & $1.95(0.21)$ & $63.37(12.44)^{\star}$ \\
YADc & 44 & 42 & $23.23(3.38)$ & $1.91(0.13)$ & $76.19(14.58)$ \\
YAD-PWR & 38 & 12 & $22.03(2.98)$ & $1.99(0.26)$ & $64.10(11.93) \ddagger$ \\
YAD-SPRJ & 21 & 21 & $21.90(2.68)$ & $2.00(0.15) \dagger$ & $61.03(7.94) \ddagger$ \\
YAD-BGP & 110 & 82 & $22.34(3.09)$ & $2.08(0.14) \ddagger$ & $58.80(10.65) \ddagger$ \\
YAD-END & 90 & 43 & $23.00(3.07)$ & $1.95(0.14)$ & $59.34(10.52)$ \\
YADa & 262 & 161 & $22.51(3.06)$ & $2.01(0.17) \ddagger$ & $59.94(10.67) \ddagger$ \\
ADc & 28 & 20 & $37.11(4.37)$ & $2.00(0.16)$ & $71.53(13.44)$ \\
ADa & 33 & 28 & $36.15(4.52)$ & $2.07(0.17)$ & $61.04(9.69) \ddagger$ \\
OADc & 11 & 9 & $50.45(4.30)$ & $2.03(0.09)$ & $70.09(9.34)$ \\
OADa & 12 & 12 & $50.25(3.05)$ & $2.00(0.10)$ & $63.83(9.70)$ \\
\hline
\end{tabular}

Values are mean (SD).

${ }^{\star} \mathrm{p}<0.05, \dagger \mathrm{p}<0.02, \neq \mathrm{p}<0.001$ compared with controls.

$\mathrm{N}$, Number of measurements; N', number of measurements in Doppler studies; CHLD, children c, control, non-trained subjects; a, athletes; AJ, adolescent/junior; YAD, young adult; AD, adult; OAD, older adult; PWR, power athletes; SPRJ, sprinters/jumpers; BGP, ball game players; END, endurance athletes.

Table 2 Echocardiographic data for young adult athletes

\begin{tabular}{lllll}
\hline & IVST (mm) & LVPWT (mm) & EDD (mm) & LVM (g) \\
\hline Control & $8.97(0.78)$ & $8.69(0.58)$ & $49.48(3.68)$ & $191.5(26.4)$ \\
PWR & $10.18(1.33)^{\star}$ & $9.84(1.37)^{\star}$ & $50.42(4.26)$ & $237.5(67.7)^{\star}$ \\
SPRJ & $10.54(0.9)^{\star}$ & $9.88(0.98)^{\star}$ & $50.56(2.89)$ & $242.4(42.3)^{\star}$ \\
BGP & $10.95(1.34)^{\star}$ & $10.54(1.35)^{\star}$ & $52.83(3.46)^{\star}$ & $280.2(60.9)^{\star}$ \\
END & $10.50(1.28)^{\star}$ & $9.91(1.11)^{\star}$ & $53.29(3.84)^{\star}$ & $264.6(50.8)^{\star}$ \\
\hline
\end{tabular}

Values are mean (SD).

${ }^{\star} \mathrm{p}<0.001$ compared with controls.

IVST, Interventricular septum thickness; LVPWT, left ventricular posterior wall thickness; EDD, left ventricular end diastolic diameter; LVM, left ventricular muscle mass; PWR, power athletes; SPRJ, sprinters/jumpers; BGP, ball game players; END, endurance athletes.

Table 3 Body size related echocardiographic data for young adult athletes

\begin{tabular}{llllll}
\hline & $\begin{array}{l}W T / B S A^{1 / 2} \\
(\mathrm{~mm} / \mathrm{m})\end{array}$ & $\begin{array}{l}E D D / B S A^{1 / 2} \\
(\mathrm{~mm} / \mathrm{m})\end{array}$ & $\begin{array}{l}L V M / B S A^{3 / 2} \\
\left(\mathrm{~g} / \mathrm{m}^{3}\right)\end{array}$ & WT/EDD (\%) & $E / A$ \\
\hline Control & $12.80(0.77)$ & $35.92(2.59)$ & $73.17(9.31)$ & $35.85(3.79)$ & $1.91(0.38)$ \\
& & & & & \\
PWR & $14.24(1.45) \dagger$ & $35.87(1.97)$ & $84.24(13.48) \dagger$ & $39.85(4.81) \dagger$ & $1.83(0.34)$ \\
SPRJ & $14.53(1.21) \dagger$ & $35.86(1.92)$ & $86.85(14.73) \dagger$ & $40.57(3.29) \dagger$ & $2.09(0.43)$ \\
BGP & $14.92(1.53) \dagger$ & $36.66(2.53)$ & $93.34(16.92) \dagger$ & $40.89(5.06) \dagger$ & $2.04(0.44)$ \\
END & $14.56(1.59) \dagger$ & $38.17(2.47) \dagger$ & $96.44(17.12) \dagger$ & $38.31(4.90)^{\star}$ & $2.21(0.62)^{\star}$ \\
\hline
\end{tabular}

Values are mean (SD).

${ }^{\star} \mathrm{p}<0.01,+\mathrm{p}<0.001$ compared with controls.

WT, Left ventricular wall thickness; BSA, body surface area; EDD, left ventricular end diastolic diameter; LVM, left ventricular muscle mass; E/A, ratio of the early and late diastolic filling peak velocity; PWR, power athletes, SPRJ, sprinters/jumpers; BGP, ball game players; END, endurance athletes.

The group of young adults was subdivided according to the type of sport. The power athletes were top level (members of national teams or first class competitors) judo competitors and weight lifters. The sprinters/jumpers group contained second class track and field athletes and top level short track skaters. The ball game players were top level and second class waterpolo and soccer players and second class handball, basketball, and volleyball players. The endurance athletes were top level road cyclists, kayak canoeists, pentathletes, and triathletes, and second class triathletes and long distance runners. Data for some athletes (fencers, gymnasts, etc) who were not classified into groups were calculated in the total.

The adult group contained several pentathletes, cyclists, and canoeists still competing at the top level and some lower level ball game players. Athletes in the older group were recreational athletes with a training regimen of a minimum of three hours and a maximum of 15 hours a week.

As Doppler examinations were not carried out on all the subjects, in the E/A quotient column of tables 3 and 5 the number of subjects examined was smaller, indicated by column $\mathrm{N}^{\prime}$ in table 1 .

Investigations were always carried out with the subject at absolute rest using a Dornier AI 4800 echocardiograph with a $2.5 \mathrm{MHZ}$ transducer. Two dimensionally guided $M$ mode recordings were obtained parasternally in accordance with the recommendations of the American Society of Echocardiography ${ }^{11}$; measurements of the left ventricular wall thickness and internal diameter were obtained by positioning the trackball cursor on the screen. All studies were performed by the same investigator (G P). Early and late diastolic peak filling velocities were estimated by pulse wave Doppler measurements in the four chamber apical view. Data were obtained across several cardiac cycles; means of five to ten cycles were used in the further analysis.

Left ventricular (LV) wall thickness (WT) was obtained as the sum of interventricular septum thickness (IVST) and posterior wall thickness (PWT). Of the several possibilities, left ventricular muscle mass (LVM) was calculated by cubing the respective diameters ${ }^{11}{ }^{12}$ as $\mathrm{LVM}=\left((\mathrm{IVST}+\mathrm{PWT}+\mathrm{EDD})^{3}-\mathrm{EDV}\right) \times$ 1.05 , where EDD is left ventricular end diastolic diameter, EDV is end diastolic volume = $\mathrm{EDD}^{3}$, and 1.05 is the density of the cardiac wall. The following exponent corrected indices were used to relate cardiac measures to body size: ' $\mathrm{WT}=\mathrm{WT} / \mathrm{BSA}^{1 / 2},{ }^{\prime} \mathrm{EDD}=\mathrm{EDD} / \mathrm{BSA}^{1 / 2}$, $\mathrm{LVM}=\mathrm{LVM} / \mathrm{BSA}^{3 / 2}$. As a relative parameter, the quotient WT/EDD was also calculated. The latter is termed relative wall thickness in many papers ${ }^{13}{ }^{14}$ and muscular quotient ${ }^{15}$ or hypertrophy index ${ }^{16}$ in others.

Mean values for athletes were compared with those for their age matched controls using $t$ tests for unpaired data. Differences at $p<0.05$ were regarded as significant.

\section{Results}

Apart from some young adult groups, there was no significant difference in body size between athletic and non-athletic groups. Resting training bradycardia was evident in all of the athletic groups, except the older adult groups where the difference was not significant (table 1).

ECHOCARDIOGRAPHIC DATA FOR YOUNG ADULT MEN COMPETING IN DIFFERENT SPORTS

Table 2 gives means of the measured values of IVST, LVPWT, and EDD and the calculated value of LVM in non-athletic subjects and different athletes, and table 3 gives the exponent corrected indices.

Absolute values of IVST, LVPWT, and LVM were significantly higher in all of the athletic groups than in the controls, but EDD was only significantly increased in ball game players and endurance athletes (table 2). No values were in 
Table 4 Echocardiographic parameters in men of different age

\begin{tabular}{lllll}
\hline & IVST (mm) & LVPWT (mm) & EDD (mm) & LVM $(\mathrm{g})$ \\
\hline CHLDc & $7.24(0.84)$ & $7.09(0.70)$ & $41.37(3.79)$ & $109.0(27.8)$ \\
CHLDa & $7.72(1.17)$ & $7.37(1.03)$ & $42.69(3.38)^{\star}$ & $123.4(33.4)$ \\
& & & & \\
AJc & $8.91(1.05)$ & $9.00(1.06)$ & $48.00(5.25)$ & $189.0(49.1)$ \\
AJa & $10.06(1.42) \dagger$ & $9.74(1.27) \dagger$ & $51.01(4.11)^{\star}$ & $238.1(63.9) \dagger$ \\
YADc & $8.97(0.78)$ & $8.68(0.58)$ & $49.43(3.68)$ & $191.5(26.4)$ \\
YADa & $10.63(1.31) \dagger$ & $10.16(1.28) \dagger$ & $52.40(3.82) \dagger$ & $264.6(59.3) \dagger$ \\
ADc & $9.36(1.26)$ & $9.03(0.87)$ & $50.70(3.67)$ & $211.3(39.1)$ \\
ADa & $10.95(1.40) \dagger$ & $10.41(1.21) \dagger$ & $51.04(3.98)$ & $261.1(55.0) \dagger$ \\
OADc & $9.95(1.23)$ & $10.03(1.86)$ & $52.86(4.43)$ & $255.2(63.9)$ \\
OADa & $10.43(1.61)$ & $10.22(1.47)$ & $51.17(4.04)$ & $252.4(60.2)$ \\
\hline
\end{tabular}

Values are mean (SD).

${ }^{\star} \mathrm{p}<0.02,+\mathrm{p}<0.001$ compared with controls

IVST, Interventricular septum thickness; LVPWT, left ventricular posterior wall thickness; EDD, left ventricular end diastolic diameter; LVM, left ventricular muscle mass; CHLD, children; c, control, non-trained subjects; a, athletes; AJ, adolescent/junior; YAD, young adult; AD, adult; $\mathrm{OAD}$, older adult.

Table 5 Body size related echocardiographic parameters in men of different age

\begin{tabular}{|c|c|c|c|c|c|}
\hline & $\begin{array}{l}W T / B S A^{1 / 2} \\
(\mathrm{~mm} / \mathrm{m})\end{array}$ & $\begin{array}{l}E D D / B S A^{1 / 2} \\
(\mathrm{~mm} / \mathrm{m})\end{array}$ & $\begin{array}{l}\operatorname{LVM} / B S A^{3 / 2} \\
\left(\mathrm{~g} / \mathrm{m}^{3}\right)\end{array}$ & $W T / E D D \%$ & $E / A$ \\
\hline CHLDc & $12.42(1.10)$ & $35.87(2.44)$ & $70.23(9.79)$ & $34.81(4.35)$ & $1.94(0.32)$ \\
\hline CHLDa & $13.28(1.57)$ & $37.64(2.21) \ddagger$ & $83.38(15.07)^{\star}$ & $35.43(4.85)$ & $2.03(0.33)$ \\
\hline $\mathrm{AJc}$ & $12.87(1.30)$ & $34.35(2.58)$ & $68.89(13.35)$ & $37.56(3.88)$ & $1.74(0.23)$ \\
\hline AJa & $14.20(1.59) \dagger$ & $36.42(2.59) \dagger$ & $86.55(17.64) \ddagger$ & $39.15(4.90)$ & $2.36(0.61) \ddagger$ \\
\hline YADc & $12.80(0.77)$ & $35.92(2.59)$ & $73.17(9.31)$ & $35.85(3.79)$ & $1.91(0.38)$ \\
\hline YADa & $14.65(1.53) \rrbracket$ & $36.99(2.54) \dagger$ & $92.36(16.86) \S$ & $39.79(4.95) \mathbb{S}$ & $2.08(0.49)^{\star}$ \\
\hline $\mathrm{ADc}$ & $13.02(1.15)$ & $35.86(2.12)$ & $74.62(10.12)$ & $36.47(4.27)$ & $1.47(0.25)$ \\
\hline $\mathrm{ADa}$ & $14.78(1.52) \rrbracket$ & $35.50(2.45)$ & $87.22(14.15) \mathbb{S}$ & $41.88(5.58) \rrbracket$ & $1.77(0.50) \dagger$ \\
\hline OADc & $14.03(1.92)$ & $37.13(2.86)$ & $88.23(21.35)$ & $37.93(5.49)$ & $1.22(0.34)$ \\
\hline OADa & $14.59(1.93)$ & $36.20(2.71)$ & $88.85(18.33)$ & $40.50(6.22)$ & $1.64(0.36) \dagger$ \\
\hline
\end{tabular}

Values are mean (SD).

${ }^{\star} \mathrm{p}<0.05,+\mathrm{p}<0.02, \neq \mathrm{p}<0.01, \S \mathrm{p}<0.001$ compared with controls.

WT, Left ventricular wall thickness; BSA, body surface area; EDD, end diastolic diameter of the left ventricle; LVM, left ventricular muscle mass; E/A, proportion of the early and late diastolic filling peak velocity; CHLD, children; c, control, non-trained subjects; a, athletes; AJ, adolescent/ junior; YAD, young adult; $\mathrm{AD}$, adult; OAD, older adult.

the pathological range, the largest IVST and LVPWT being 15.30 and $14.36 \mathrm{~mm}$ respectively.

Using the exponent corrected indices (table 3), athletic groups displayed increased LV measures, but the two components of hypertrophy varied. A highly significant increase was seen in the 'WT in all groups, with no appreciable difference among the athletic groups. 'EDD, however, was significantly larger only in endurance athletes; in the ball game players it tended to be only slightly larger (not significant).

'LVM showed a highly significant positive difference for all the athletic groups; groups could be ranked in a consistently increasing order of power athletes, sprinters/jumpers, ball game players, endurance athletes.

Because in LV hypertrophy WT had a more pronounced role than EDD, the ratio of these two variables was high in all of the athletic groups, there being no obvious difference between the different branches. Slightly higher values were found in the ball game players, and the lowest ones were found in the endurance athletes.

The E/A quotient (ratio of peak velocity during early and late diastole) was significantly higher in the endurance athletes only. In the sprinters/jumpers and ball game players, a slight tendency to increase was seen.

ECHOCARDIOGRAPHIC PARAMETERS AT DIFFERENT AGES

Table 4 gives the means of the measured values of IVST, LVPWT, and EDD and the calculated value of LVM in non-athletic and athletic groups of different age, and table 5 gives the exponent corrected indices.

Significant differences were seen between the athletic and non-athletic groups in IVST, LVPWT, and LVM in the adolescent, young adult, and adult groups, while, for EDD, intergroup differences were significant only for the children, adolescents, and young adults (table 4).

The morphological cardiac dimensions related to the exponent corrected body size measure (table 5) were very similar in the control subjects of different age; only the oldest group seemed to have a slightly higher 'WT and 'LVM.

Concerning training induced LV hypertrophy, however, some differences were observed in both its extent and the share of its two components at the different ages. In the athletic children, 'EDD was significantly higher than in the non-athletic ones, and 'WT was slightly but not significantly larger. In the adolescent/junior groups, the difference in 'WT reached significance. In young adults, differences in 'WT were more pronounced than in 'EDD. In the adult groups, hypertrophy was only manifest in 'WT, and significant differences were absent in the older adults. As a result, differences in 'LVM increased from those in children $(18.7 \%)$ to those in adolescent/juniors and young adults (25.6\% and $26.2 \%$ respectively), then decreased in the adult group (16.9\%), and had disappeared in the older adults. WT/EDD was not significantly different in the children and in the adolescent/junior group; the difference from controls was significant in the young adult group $(11.0 \%)$, reached a maximum in the $31-44$ year age group (39.8\%), and disappeared again in the older groups.

Except for in children, the E/A quotient was significantly higher in the athletic groups at all ages. It should be noted that differences between the three adult age groups (young adults, $8.9 \%$; adults, $20.4 \%$; older adults $34.4 \%$ ) consistently increased (table 5).

\section{Discussion}

In our recent reports ${ }^{12}$ and one from another group, ${ }^{16}$ it has been shown that heart indices in which the dimensional exponents of numerator and denominator match do not correlate with body size whereas simple BSA related indices do. The corrected indices would seem to be more suitable for comparing subjects of different body size..$^{1-4}$ In our material-for example, the values of non-athletic control subjects of different age proving to be very similar-the slightly thicker left ventricular wall in the older adults may actually have been an age dependent hypertrophy, partly caused by slightly higher blood pressure (141.4 (14.6)/94.55 
(8.79) $\mathrm{mm} \mathrm{Hg}$ ). In this study, the results of 578 male subjects were analysed using this correction.

Our observations on the different types of sport played by young adults (19-30 years) were very similar to the results of other publications in which a large amount of data on different athletes were also compared. ${ }^{17-21}$

'LVM, the most consistent determinant of training induced hypertrophy, ranked the subjects in the order non-athletes, power athletes, sprinters/jumpers, ball game players, endurance athletes. This was attributable to exercise induced differences in 'EDD rather than 'WT which had a consistently higher value in all competitive groups.

Our results agree with reports ${ }^{13}{ }^{18} 22-25$ indicating that concentric and eccentric hypertrophy cannot be clearly distinguished in training induced myocardial hypertrophy. WT/EDD and 'WT were not the highest in the power athletes, indeed, the latter was found to be slightly lower than in any of the other athletic groups. It cannot be ruled out, however, that the power athletes investigated here had a relatively short training history, not long enough to develop pronounced thickening of the LV wall. In fact, of the athletic groups, the endurance athletes had the lowest WT/EDD, but even they had values that greatly exceeded those of the non-athletes.

It seems best not to categorise by using concentric and eccentric hypertrophy, but to state that an increase in WT can be expected with any kind of regular physical training, while an increase in EDD seems to be caused predominantly by endurance activity.

Several reports deal with data on children, adolescents, and/or young athletes. ${ }^{26-30}$ Few of them, however, compare young, adult, and older athletes with respect to training induced modifications. Our data indicate that an increase in 'LVM is already apparent in childhood; it reaches near maximal values at the adolescent/junior age, and remains maximal in young adults. In the younger groups, 'WT shows a smaller increase and 'EDD shows a more pronounced increase than in adult athletes, and therefore WT/EDD remains unchanged. A significant increase in this ratio is first observed in the young adult groups. This difference can be explained by the classic observation that regular physical training first induces ventricular dilatation, and muscular hypertrophy only begins to develop some time later.

Immediately after the competitive age range, training induced modifications in the structure and function of the heart seem to remain significant, and only the extent of the difference tends to decrease.

At an older age (45-60 years), morphological differences are likely to disappear, mostly as the result of an increase in 'WT even in untrained subjects. Morphological cardiac changes in the older group need a more detailed analysis, especially as few older athletes are engaged in such extensive and intense physical training as is usual at a younger age. This more detailed analysis should also be extended to the functional characteristics of the heart.

The present results show, however, that 'LVM hypertrophy and increased WT/EDD ratio and 'WT can no longer be regarded as unambiguous signs of an athletic heart. To characterise the condition of the heart, it is useful to estimate some functional signs, primarily the E/A quotient, which indicates diastolic function.

Two main questions arise with respect to the E/A quotient. Does regular physical training cause an increase in normal values in the young ${ }^{31-35}$ or not ${ }^{23}{ }^{36-39}$ ? Is regular physical training able to prevent the age dependent impairment of left ventricular diastolic function ${ }^{40-42}$ or not? ${ }^{43} 44$

In answer to both questions, our results support a beneficial effect of regular physical training. However, the observations on the young adult athletes show that the type of physical training has an important role. No increase was found in the strength athletes, whereas, in the other groups, there was a more or less pronounced increase, indicating that dynamic, mostly endurance-type, training appears to be necessary to elicit a higher compliance of the left ventricle. This inference is in accordance with most other data: Pearson et $a l^{23}$ found no increase in weight lifters, and, in the studies reporting a positive effect of physical training, the athletes were mostly of the endurance type. ${ }^{32} 3335$

It is obvious that a few years of training is not enough to induce such modifications, and that is why there is no difference in childhood. In the 15-18 year and 19-30 year groups, however, an increase in the $\mathrm{E} / \mathrm{A}$ quotient was evident.

With respect to public health, the most important fact seems to be that the E/A quotient was much higher in the older athletes than in the non-athletic subjects. Thus, our results support the suggestion that regular physical training may prevent age dependent impairment of left ventricular compliance. ${ }^{40-42}$ It is difficult to explain why other authors ${ }^{43} 44$ have failed to find any beneficial effect. Obviously, the results of such investigations depend on several factors, including the exact age of the subjects and the volume and intensity of the activity. As our older athletes had mostly performed at the top level in their youth, we suggest that a more effective positive influence of regular physical training on diastolic function can be expected when athletic conditioning is sufficiently intense at a younger age and has been continuously maintained throughout the years.

This research was supported by the World Bank grant IFB:478.

1 Pavlik G, Olexó Zs, Petrekanits M, et al. Arithmetic calculation with echocardiographic indices (in Hungarian, abstract in English). Hungarian Review of Sports Medicine 1995;36:115-29.

2 Pavlik G, Olexó Zs, Frenkl R. Echocardiographic estimates related to various body size measures in athletes. Acta Physiol Hung 1996;84:171-81.

3 George KP, Gates PE, Birch KM, et al. Left ventricular morphology and function in endurance-trained female athletes. $\mathcal{F}$ Sports Sci 1999;17:633-42. 
4 George KP, Gates PE, Whyte G, et al. Echocardiographic examination of cardiac structure and function in elite cross trained male

5 Bryg RJ, Williams GA, Labowitz AJ. Effect of aging on left ventricular diastolic filling in normal subjects. Am $\mathcal{F}$ Cardio 1987; 59:971-4.

6 Gardin JM, Henry WL, Savage DD, et al. Echocardiographic measurements in normal subjects: evaluation of an adult population without clinically apparent heart disease. f Clin Ultrasound 1979;7:439-47.

7 Gerstenblith G, Frederiksen J, Yin FCP, et al. Echocardiographic assessment of a normal adult aging population. Circulation 1977;56:273-8.

8 Longhurst JC, Kelly AR, Gonyea WJ, et al. Cardiovascular responses to static exercise in distance runners and weight lifters. F Appl Physiol 1980;49:676-83.

9 Sartori MP, Quinones MA, Kuo LC. Relation of Dopplerderived left ventricular filling parameters to age and radius/ thickness ratio in normal and pathological states. $A m f$ thickness ratio in norma

10 Szlachcic T, Tubau JF, O'Kelly B, et al. Correlates of diastolic filling abnormalities in hypertension: a doppler echocardiographic study. Am Heart f 1990;120:386-91.

11 Sahn DJ, De Maria A, Kisslo J, et al. Recommendations regarding quantitation in M-mode echocardiography. Results of a survey of echocardiographic measurements. Circulation 1978;58:1072-83.

12 Henry WL, De Maria A, Gramiak R, et al. Report of the American Society of Echocardiography: nomenclature and standards in two-dimensional echocardiography. Circulation 1980;62:212-17.

13 Fagard RH. Athlete's heart: a meta-analysis of the echocardiographic experience. Int f Sports Med 1996;17:S140-4.

14 Douglas PS, O'Toole ML, Katz SE, et al. Left ventricula hypertrophy in athletes. Am F Cardiol 1997;80:1384-8.

15 Dickhuth HH, Simon G, Schmied P, et al. Blutdruckverhalten und kardiale Anpassungserscheinungen bei Hochleisalten und kardiale Anpassungserscheinungen bei Hoch

16 Urhausen A, Monz T, Kindermann W. Sports-specific adaptation of left ventricular muscle mass in athlete's heart I. An echocardiographic study with combined isometric I. An echocardiographic study with combined isometric
and dynamic exercise trained athletes (male and female

17 Dickhuth HH, Simon G, Kindermann W, et al. Echokardiographische Untersuchungen bei Sportlern verschiedener Sportarten und Untrainierten. Z Kardiol 1979;68:449-53.

18 Pelliccia A. Determinants of morphologic cardiac adaptation in elite athletes: the role of athletic training and constitutional factors. Int F Sports Med 1996;17:S157-63.

19 Spataro A, Pelliccia A, Caselli G, et al. Echocardiographic standards in top-class athletes. F Sports Cardiol 1985;2:1727.

20 Spirito P, Pelliccia A, Proschan MA, et al. Morphology of the "athletes heart" assessed by echocardiography in 947 elite athletes representing 27 sports. Am F Cardiol 1994;74:8026.

21 Zott H-J, Kästner C. Kardiologische Ultraschalldiagnistik in der Sportmedizin. Leipzig: Johann Ambrosius Barth, 1991.

22 Fagard RH, Aubert A, Lysens R, et al. Noninvasive assessment of seasonal variations in cardiac structure and

23 Pearson AC, Schiff M, Mrosek D, et al. Left ventricular diastolic function in weight lifters. Am F Cardiol 1986;58: 1254-9.

24 Pelliccia A, Maron BJ. Outer limits of the athlete's heart, the effect of gender, and relevance to the differential diagnosis with primary cardiac diseases. Cardiol Clin 1997;15:38196.
25 Pelliccia A, Spataro A, Caselli G, et al. Absence of left ventricular wall thickening in athletes engaged in intense power training. Am F Cardiol 1993; 72:1048-54

26 Csanády $M$, Forster T, Hõgye $M$. Comparative echocardiographic study of junior and senior basketball players. Int $\mathcal{F}$ Sports Med 1986;7:128-32.

27 Csanády $M$, Forster T, Hõgye $M$, et al. Three year echocardiographic follow-up study on canoeist boys. Acta Cardiol 1986;41:413-25.

28 Mesko D, Jurko A, Vrlík M, et al. Development of the left ventricular hypertrophy and dilation in adolescent ice hockey players evaluated with echocardiography. Sports Medicine and Training Rehabilitation 1993;4:177-88.

29 Pavlik G, Simon Gy, Olexó Zs, et al. Cardiac and spiroergographic indices in young football players (in Hungarian, abstract in English). Hungarian Review of Sports Medicine 1993;34:17-31.

30 Rost R. Das Herz des Sportlers im Ultraschall. Schorndorf: Karl Hofmann, 1982.

31 Matsuda M, Sugishita Y, Koseki S, et al. Effect of exercise on left ventricular diastolic filling in athletes and nonathletes. F Appl Physiol 1983;55:323-8.

32 Finkelhor RS, Hanak LJ, Behler RC. Left ventricular filling in endurance-trained subjects. $\mathcal{F}$ Am Coll Cardiol 1986;8: 289-93.

33 Douglas PS, O'Toole ML, Hiller DB, et al. Left ventricular structure and function by echocardiography in ultraendurance athletes. Am f Cardiol 1986;58:805-9.

34 Colan SD, Sanders SP, MacPherson D, et al. Left ventricular diastolic function in elite athletes with physiologic cardiac hypertrophy. F Am Coll Cardiol 1985;6:545-9.

35 Möckel M, Störk T. Diastolic function in various forms of left ventricular hypertrophy: contribution of active Doppler stress echo. Int $\mathcal{F}$ Sports Med 1996;17:S184-90.

36 Fagard RH, van den Broeke C, Bielen E, et al. Assessment of stiffness of the hypertrophied left ventricle of bicyclists using left ventricular inflow Doppler velocitometry. $7 \mathrm{Am}$ Coll Cardiol 1987;9:1250-4.

37 Shapiro LM, Smith RG: Effect of training on left ventricular structure and function: an echocardiographic study. $\mathrm{Br}$ Heart $\mathcal{F}$ 1983;50:534-9.

38 Granger CB, Karimeddini MK, SmithVE, et al. Rapid ventricular filling in left ventricular hypertrophy. I. Physiologic hypertrophy. F Am Cell Cardiol 1985;5:862-8.

39 Missault L, Duprez D, Jordaens L, et al. Cardiac anatomy and diastolic filling in professional road cyclists. Eur $\mathcal{F} A p p l$ Physiol 1993;66:405-8.

40 Takemoto KA, Bernstein L, Lopez JF, et al. Abnormalities of diastolic filling of the left ventricle associated with aging are ess pronounced in exercise trained individuals. Am Heart 7 1992;124:143-8.

41 Douglas PS, O'Toole M. Aging and physical activity determine cardiac structure and function in the older athlete. $\mathcal{F}$ Appl Physiol 1992;72:1969-73.

42 Levy WC, Cerqueira MD, Abrass IB, et al. Endurance exercise training augments diastolic filling at rest and during exercise in healthy young and older men. Circulation 1993; 88:116-26.

43 Schulman SP, Lakatta EG, Fleg JL, et al. Age-related decline in left ventricular filling at rest and exercise. Am 7 Physiol 1992;263:H1932-8

44 Fleg JL, Shapiro EP, O'Connor F, et al. Left ventricular diastolic filling performance in older male athletes. $f A M A$ 1995;273:1371-5

Take home message

Numbers can lead and mislead, depending on how you use them! 